\title{
Refractory headaches treated with bilateral occipital and temporal region stimulation
}

\author{
This article was published in the following Dove Press journal: \\ Medical Devices: Evidence and Research \\ 25 March 2014 \\ Number of times this article has been viewed
}

\author{
Kelly J Zach' \\ Terrence L Trentman' \\ Richard S Zimmerman ${ }^{2}$ \\ David W Dodick ${ }^{3}$ \\ 'Department of Anesthesiology, \\ ${ }^{2}$ Department of Neurosurgery, \\ ${ }^{3}$ Department of Neurology, Mayo \\ Clinic in Arizona, Phoenix, AZ, USA
}

Correspondence: Terrence L Trentman Department of Anesthesiology, Mayo Clinic in Arizona, 5777 E Mayo Blvd, Phoenix, AZ 85054, USA

Tel + I 480342 I800

$\mathrm{Fax}+\mathrm{I} 4803422319$

Email trentman.terrence@mayo.edu
Objectives: To describe use of bilateral temporal and occipital stimulator leads for a refractory headache disorder.

Materials and methods: A 31-year-old female had a 10-year history of chronic, severe occipital and temporal region headaches. The patient underwent permanent implant of an occipital stimulator system that resulted in sustained, compete resolution of her occipital pain. However, she continued to suffer disabling (10/10) temporal region headaches and was bedbound most days of the week. Therefore, bilateral temporal stimulator leads were implanted and tunneled to her internal pulse generator.

Results: At 12-month follow-up, the patient enjoyed sustained improvement in her pain scores $(8 / 10)$ and marked increase in her level of functioning. Taking into account increased activity level, she rated her overall improvement at 50\%. Unfortunately, infection and erosion of her right temporal lead necessitated temporal stimulator removal.

Conclusion: Headache disorders may require stimulation of all painful cephalic regions. However, our success in this case must be considered in light of the technical challenges and expense of placing stimulator leads subcutaneously around the head and neck, including the risk of infection, lead breakage, erosion, and migration.

Keywords: headache, occipital nerve stimulation, peripheral nerve stimulation, migraine, cluster headache

\section{Introduction}

Peripheral nerve stimulation (PNS) has potential benefits in a variety of refractory headache disorders including chronic migraine, occipitocervical headaches, chronic cluster headache, and hemicrania continua. ${ }^{1}$ Clinicians have demonstrated progress in utilizing PNS for chronic pain disorders, including lower back, groin, and thoracic pain. $^{2}$ Over the last decade, a growing body of literature also suggests efficacy of PNS for headaches. ${ }^{3,4}$ Headache disorders affect nearly $50 \%$ of the global population. ${ }^{5}$ Initial treatment options for controlling headaches include pharmacologic agents, nerve blocks, physical therapy and biofeedback. However, these methods may produce limited or temporary relief. PNS is a minimally invasive alternative for refractory primary headache disorders.

In 1999, Weiner and Reed successfully treated a series of patients with occipital pain by stimulating the occipital nerves (ONS). ${ }^{6}$ Later, a patient with chronic cluster headache had complete remission of pain after stimulation of the supraorbital nerve. ${ }^{7}$ Recently, combined occipital and supraorbital stimulation yielded a better response than occipital nerve stimulation alone in a group of patients with holocephalic pain. ${ }^{8}$ 
These initial successes of PNS for refractory headache disorders suggest opportunities for additional, site-specific stimulation techniques. Herein, we describe bilateral temporal region stimulation combined with bilateral occipital stimulation in a patient with intractable and debilitating temporal and occipital headaches.

\section{Case report}

A 31-year-old female had a 10-year history of daily severe occipital and temporal region headaches that were refractory to numerous conservative therapies. She had initially presented with new daily persistent headache (NPDH) in the absence of a prior history of migraine. However, the phenotype of the headache disorder did not meet ICHD-II (International Headache Classification - 2nd edition) criteria for NDPH due to the daily presence of migraine features including nausea, photophobia, and phonophobia. The phenotype did meet the ICHD-IIR (revised) criteria for chronic migraine, with the exception of not having 5 prior attacks. A complete, thorough, and exhaustive search for secondary causes was unrevealing. The patient developed a daily and persistent headache in the absence of any analgesic use; ie, she did not have a medication overuse headache. She did begin to overuse analgesic medications as a consequence of her new and persistent daily pain. The patient was withdrawn from all analgesics after a rigorous withdrawal schedule. Unfortunately, despite being off analgesics for $>3$ months, there was no change in her headache pattern in terms of severity, disability, or its continuous nature.

The patient was ultimately diagnosed with chronic migraine $(>15$ headache days per month for at least 3 months) and treated with numerous medications including divalproex sodium, amitriptyline, gabapentin, topiramate, botulinum toxin, opiates, and metoclopramide. Lithium and verapamil were also tried after all other conventional and evidence-based options had been exhausted.

The patient was referred to the pain clinic and after a 5 day successful trial ( $>50 \%$ reduction in pain severity) of bilateral ONS with Advanced Neuromodulation Systems leads (now St Jude Medical Neuromodulation; St Paul, MN, USA) the patient underwent permanent implant of an ONS system that included bilateral quadripolar leads and placement of an internal pulse generator (IPG) in her lower lateral abdomen. This resulted in sustained, compete resolution of her occipital pain. However, she continued to report significant pain in her temporal regions bilaterally that was unfortunately not diminished with ONS. Specifically, she identified the areas lateral to the eyebrows and superior to her zygomatic arches as the regions most affected.

Despite medical management, the patient experienced daily pain in these areas. She reported to her headache neurologist that her bilateral temporal pain was 10/10 in intensity, and that four to five days per week she was bedbound. Her primary care physician prescribed local anesthetics that she self-injected subcutaneously up to eight times per day, providing mild relief from her intractable temporal headaches. After consultation with neurology headache, pain management, and neurosurgical specialists at our institution, the interdisciplinary team felt she would benefit from placement of bilateral temporal region stimulators.

Under intravenous sedation and local anesthesia, a St Jude Neuromodulation quadripolar lead (St Jude Medical Neuromodulation) was inserted subcutaneously from a point approximately $6 \mathrm{~cm}$ above the temporal region. This was carried out bilaterally through a 14-gauge Tuohy needle, with the goal of maintaining the needle in the subcutaneous fat, above the fascial layer. Intraoperative testing confirmed appropriate patterns of stimulation in the region of the patient's temporal pain. The stimulation patterns were a result of various electrode combinations, rates, and pulse widths that provided a non-painful sensation of stimulation.

Next, under general anesthesia, the left electrode was tunneled to a point near the vertex in the subcutaneous channel. Both leads were then tunneled down the right side of her neck and between her breasts to reach the IPG (Figure 1). Therefore, the patient had tunneled extensions both anteriorly for her temporal stimulator and posteriorly for her ONS (Figure 2).

The patient tolerated the procedure well. At 6-month and 12-month follow up, the patient noted considerable improvement in her daily headaches. Stimulation settings were adjusted at each follow-up visit to patient comfort and effect, and varied somewhat over time. Her stimulator

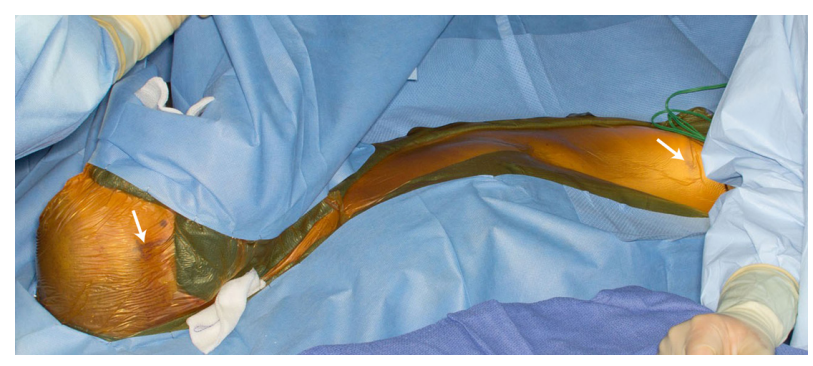

Figure I Intraoperative photograph demonstrates draping for bilateral temporal lead placement with subsequent tunneling via the patient's right neck, chest, and abdomen. Arrows point to the site of the temporal lead and the Internal Pulse Generator. 


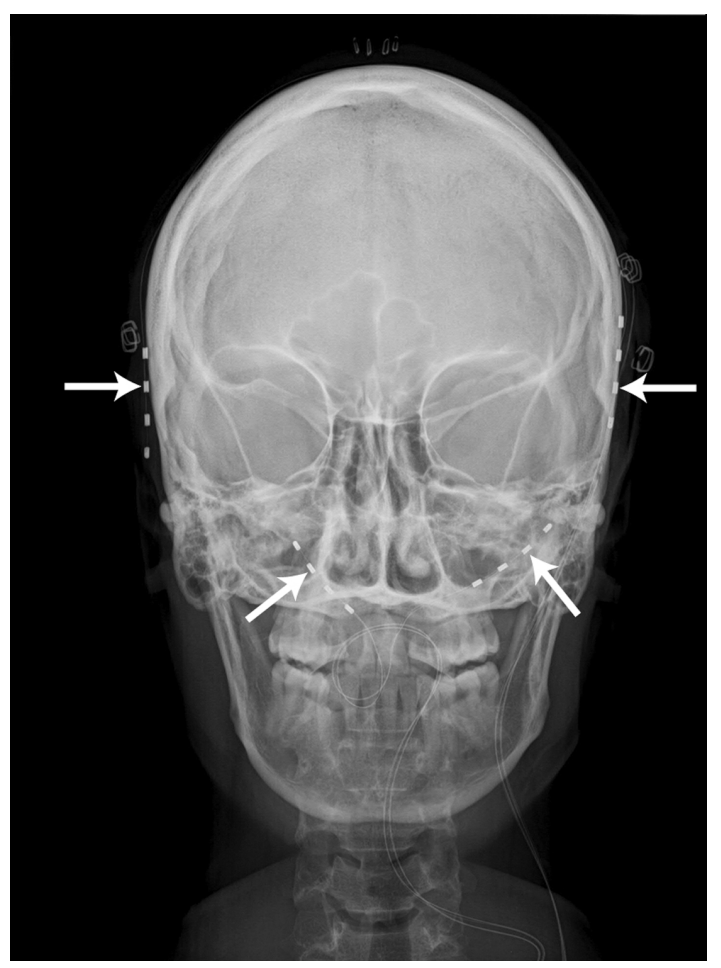

Figure 2 Postoperative X-ray demonstrates bilateral temporal and occipital stimulator leads. The four arrows point to the temporal and occipital leads.

settings fell into ranges typical for this patient population: rate of 30-60 Hz; amplitude 1-5 volts; pulse width 250-450 milliseconds. ${ }^{9}$

The patient essentially began to experience improvement immediately after implantation. Prior to implantation, she reported daily 10/10 pain; following the operation, she was reporting 10/10 temporal pain just one day per week and on average, $8 / 10$ on the numeric pain scale, which she considered a significant improvement although she continued to have daily headaches. She was using both the occipital and temporal stimulators continuously and her only pain medications were over-the-counter analgesics on a pro re nata basis. Overall, she noted a 50\% improvement in her functional status. She was no longer bedbound the majority of the week; she was able to participate in activities of daily living including caring for her three-year-old child and attending yoga classes. Her quality of life was markedly improved and during the intervening year she delivered a second child.

Unfortunately, an infection developed over the anchor in her right parietal region. Initially, antibiotic treatment was attempted but when component erosion occurred, the bi-temporal stimulator components were removed. The patient has indicated she strongly wants the temporal stimulator replaced as soon as possible.

\section{Discussion}

This case report describes successful treatment of a complex, severe headache disorder that included both occipital and temporal region pain. Although we have 12 month follow-up supporting an excellent outcome, the patient's course was complicated by an infection and erosion, necessitating explantation of the temporal stimulator system.

Many headache sufferers describe pain in multiple head regions, and ONS has been successful in some of these patients with more diffuse cephalgia. However, as was seen in our patient, stimulation that covers all painful areas may be necessary. Pain that is anterior to the vertex may not respond as well to ONS as pain that involves the occipital and suboccipital region. Despite the anatomical and functional convergence of the trigeminal and cervical afferents within the trigeminocervical complex, and despite our expectation therefore that anterior pain would respond to occipital stimulation, this does not appear to be the case in a significant proportion of patients.

The mechanism of occipital stimulation and peripheral nerve (field) stimulation of the head is incompletely understood. Just as stimulating the occipital nerves may influence the activity of sensory neurons in the trigeminocervical complex, stimulating the trigeminal nerve may do the same. It's also possible that a broader distribution of stimulation is better able to activate endogenous descending pain modulation pathways. Recent animal evidence indicates that trigeminal nerve branches in the temporalis region are contiguous with dural sensory fibers and share the same mechanical receptive field. Therefore, it is plausible that extracranially stimulating trigeminal fibers in the temporal region directly modulates the activity of dural sensory fibers. ${ }^{10}$

Headache disorders produce significant individual suffering and an enormous economic burden on the patients, insurers, and government agencies. Annual direct costs for chronic migraine are estimated to be $\$ 4,144$ per patient in the US. ${ }^{11}$ Many patients that suffer headaches are resistant to standard treatments including pharmacotherapy and nerve blocks. Studies show a prevalence of chronic migraine of $0.9 \%-5.1 \%,{ }^{12}$ despite multimodal therapeutic approaches. Patients with refractory headaches might benefit from more advanced therapies, such as peripheral nerve stimulation.

Over the last decade, the use of peripheral nerve stimulation for treating headaches has expanded and evolved. Roughly 1,200 patients have been treated with nerve stimulation for intractable headaches. Successful outcomes with the use of PNS have been documented in a variety of studies including high quality, controlled trials. ${ }^{13}$ In a randomized controlled 
study by Silberstein et $\mathrm{al}^{14}$ the primary endpoint (percent of patients with $\geq 50 \%$ reduction in headache pain) was not met, but patients treated with ONS showed clinically significant reductions in headache days, and migraine-related disability. Furthermore, stimulation of distal trigeminal branches including supraorbital nerve stimulation has been described. ${ }^{15}$

Success of ONS for migraine headaches has led to the site-specific stimulation of headaches covering other anatomic regions, although descriptions of this concept in the literature are limited..$^{7,15,16}$ The findings of Reed et $\mathrm{al}^{8}$ suggest improved management of headaches using combined ONS plus supraorbital stimulation versus ONS alone; Deshpande and Wininger ${ }^{17}$ used combined ONS and temporal region stimulation for a patient with occipital neuralgia and migraine; and Mammis et al ${ }^{18}$ described cluster headache management with ONS plus supra- and infraorbital stimulation. Our success in utilizing bilateral temporal stimulators to treat bitemporal pain adds to this body of experience on the use of PNS for chronic, intractable headaches. Furthermore, it suggests site-specific stimulation may be needed in some patients.

Balanced against this success are the considerable financial costs for stimulation and technical challenges of placing stimulator leads subcutaneously around the head and neck, and the risk of infection and component erosion. Lead migration remains a risk of ONS and any lead that traverses highly mobile body regions including the head and neck. Our patient was particularly challenging due to her lower lateral abdominal IPG and the need to tunnel extensions both front and back. A recent technical note discusses a surgical technique to minimize disadvantages of peripheral nerve stimulation for headaches such as infectious risks, lead migration, and unsatisfactory cosmetic outcome, ${ }^{19}$ and another report discusses ultrasound guidance for ONS lead placement. ${ }^{20}$

\section{Conclusion}

In conclusion, this case report describes a patient with intractable bilateral temporal headaches resulting in severe disability and poor quality of life. She had previously undergone placement of an ONS system with complete resolution of occipital pain; we subsequently placed bilateral temporal stimulators to treat the pain in those regions. After a year, the patient reported a 50\% overall improvement; unfortunately, her course was complicated by an infection requiring explantation of the temporal leads. This case illustrates the success of implanted stimulators for headaches but also complications that can be costly and time consuming.
Formal studies of site-specific headache stimulation are indicated, including analysis of cost and outcome.

\section{Author contributions}

All authors collected and analyzed information for the report, and contributed to preparation of the manuscript. All authors reviewed the manuscript critically and approved the submitted version of the manuscript. The authors agree to be accountable for all aspects of the work and will ensure that questions related to the accuracy or integrity of any part of the work are appropriately investigated and resolved.

\section{Disclosure}

Within the past 5 years, Drs Trentman and Dodick have received research support from St Jude-Neuromodulation and Medtronic Inc. Dr Dodick has provided consulting services within the past 3 years for Medtronic, Inc., and Boston Scientific Corporation. In the remote past ( $>3$ years), Drs Trentman and Zimmerman provided consulting services for Advanced Bionics Corporation, now Boston Scientific Neuromodulation. The authors declare that no financial support was given towards the preparation of this manuscript.

\section{References}

1. Brewer AC, Trentman TL, Ivancic MG, et al. Long-Term outcome in occipital nerve stimulation patients with medically intractable primary headache disorders. Neuromodulation. 2013;16(6):557-564.

2. Aló KM, Abramova MV, Richter EO. Percutaneous peripheral nerve stimulation. Prog Neurol Surg. 2011;24:41-57.

3. Weiner RL, Aló KM. Occipital Neurostimulation for Treatment of Intractable Headache Syndromes. In: Krames ES, Peckham PH, Rezai AR, editors. Neuromodulation. Vol 1 and 2. Waltham: Academic Press; 2009:409-416.

4. Freeman JA, Trentman TL. Clinical utility of implantable neurostimulation devices in the treatment of chronic migraine. Med Devices (Auckl). 2013;6:195-201.

5. Stovner Lj, Hagen K, Jensen R, et al. The global burden of headache: a documentation of headache prevalence and disability worldwide. Cephalalgia. 2007;27(3):193-210.

6. Weiner RL, Reed KL. Peripheral neurostimulation for the control of intractable occipital neuralgia. Neuromodulation. 1999;2(3):217-221.

7. Narouze SN, Kapural L. Supraorbital nerve electric stimulation for the treatment of intractable chronic cluster headache: a case report. Headache. 2007;47(7):1100-1102.

8. Reed KL, Black SB, Banta CJ 2nd, Will KR. Combined occipital and supraorbital neurostimulation for the treatment of chronic migraine headaches: initial experience. Cephalalgia. 2010;30(3):260-271.

9. Trentman TL, Zimmerman RS, Seth N, Hentz JG, Dodick DW. Stimulation ranges, usage ranges, and paresthesia mapping during occipital nerve stimulation. Neuromodulation. 2008;11(1):56-61.

10. Schueler M, Messlinger K, Dux M, Neuhuber WL, De Col R. Extracranial projections of meningeal afferents and their impact on meningeal nociception and headache. Pain. 2013;154(9):1622-1631.

11. Lanteri-Minet M. Economic burden and costs of chronic migraine. Curr Pain Headache Rep. 2014;18(1):385.

12. Natoli JL, Manack A, Dean B, et al. Global prevalence of chronic migraine: a systematic review. Cephalalgia. 2010;30(5):599-609. 
13. Tavanaiepour D, Levy RM. Peripheral neuromodulation for treatment of chronic migraine headache. Journal of Neurosurgical Review. 2013;2:1-4.

14. Silberstein SD, Dodick DW, Saper J, et al. Safety and efficacy of peripheral nerve stimulation of the occipital nerves for the management of chronic migraine: results from a randomized, multicenter, doubleblinded, controlled study. Cephalalgia. 2012;32(16):1165-1179.

15. Slavin KV, Colpan ME, Munawar N, Wess C, Nersesyan H. Trigeminal and occipital peripheral nerve stimulation for craniofacial pain: a singleinstitution experience and review of the literature. Neurosurg Focus. 2006;21(6):E5

16. Amin S, Buvanendran A, Park KS, Kroin JS, Moric M. Peripheral nerve stimulator for the treatment of supraorbital neuralgia: a retrospective case series. Cephalalgia. 2008;28(4):355-359.
17. Deshpande KK, Wininger KL. Feasibility of combined epicranial temporal and occipital neurostimulation: treatment of a challenging case of headache. Pain Physician. 2011;14(1):37-44.

18. Mammis A, Gudesblatt M, Mogilner AY. Peripheral neurostimulation for the treatment of refractory cluster headache, long-term follow-up: case report. Neuromodulation. 2011;14(5):432-435; discussion 435.

19. Skaribas IM, Aló KM. Technical Note: Optimizing Treatment for Holocephalic Neuralgia with Combined Bilateral Supraorbital and Occipital Neurostimulation Electrodes and an Internal Pulse Generator. Journal of Neurosurgical Review. 2013;2:5-10.

20. Skaribas I, Aló K. Ultrasound imaging and occipital nerve stimulation. Neuromodulation. 2010;13(2):126-130.

\section{Publish your work in this journal}

Medical Devices: Evidence and Research is an international, peerreviewed, open access journal that focuses on the evidence, technology, research, and expert opinion supporting the use and application of medical devices in the diagnosis, treatment and management of clinical conditions and physiological processes. The identification of novel devices and optimal use of existing devices which will lead to improved clinical outcomes and more effective patient management and safety is a key feature. The manuscript management system is completely online and includes a quick and fair peer-review system. Visit http://www. dovepress.com/testimonials.php to read real quotes from authors.

Submit your manuscript here: http://www.dovepress.com/medical-devices-evidence-and-research-journal 